\title{
Une nouvelle famille de Nématodes parasites d'Invertébrés marins, les Benthimermithidae (1)
}

\author{
par Annie-J. PETTER \\ Laboratoire des Vers, associé au C.N.R.S., \\ Muséum national d'Histoire naturelle, 43, rue Cuvier, F 75231 Paris Cedex 05
}

RESUME. Des Nématodes appartenant à un groupe apparenté aux Mermithoidea et connu seulement par une note préliminaire de Hope (1977) ont été trouvés, soit parasites d'Invertébrés (uniquement femelles juvéniles), soit libres (femelle mûre et femelle juvénile), dans des sédiments benthiques de l'Océan Atlantique, de la Mer de Norvège et de l'Océan Indien.

Les caractères communs à l'ensemble du groupe sont donnés. Benthimermis hopei, n. gen., n. sp., connue par une femelle mature et une femelle juvénile, trouvées libres dans des sédiments benthiques de l'Océan Atlantique, est décrite, ainsi que 8 femelles juvéniles (Benthimermis sp., $\mathrm{n}^{\text {os }} 1$ à 8 ), dont 7 sont parasites d'Invertébrés et une a été trouvée libre dans les sédiments.

Une nouvelle famille est créée pour le groupe, les Benthimermithidae, caractérisée par un œsophage vestigial, réduit à un cordon à contenu granuleux, un intestin transformé en trophosome et un rectum et un anus vestigiaux.

\section{A new family of Nematodes parasites of marine Invertebrates, the Benthimer- mithidae.}

SUMMARY. Nematodes of a group related to the Mermithoidea and known only from a preliminary note by Hope (1977) have been found either as parasites of invertebrates (only female juveniles) or free-living (mature and juvenile females) in benthic sediments of the Atlantic Ocean, the Norway Sea, and the Indian Ocean.

The characters in common in the group are given. Benthimermis hopei, n. g., n. sp., known from one mature and one juvenile females found free in benthic sediments of the Atlantic Ocean is described, as well as 8 juvenile females (Benthimermis sp., $\left.n^{\text {os }} 1-8\right)$, of which 7 are parasites of invertebrates and one was found free in sediments. A new family is proposed for the group, the Benthimermithidae, characterized by $a$ vestigial oesophagus, reduced to a strand containing granular material, an intestine modified into a trophosome, and a vestigial rectum and anus.

(1) Le matériel faisant l'objet de cette étude a été trié par le Centre national de Tri d’Océanographie Biologique (CENTOB).

Accepté le 20 novembre 1979. 
Nous avons été chargée par le CENTOB d'examiner des Nématodes parasites d'Invertébrés marins (principalement Crustacés), provenant de sédiments benthiques prélevés au cours de plusieurs campagnes effectuées dans l’Océan Atlantique, la Mer de Norvège et l'Océan Indien; nous avons constaté qu'il s'agissait de femelles juvéniles appartenant à un groupe encore à peu près inconnu, apparenté aux Mermithoidea.

Ultérieurement, nous avons trouvé dans un lot de Nématodes libres provenant également de sédiments benthiques de l'Océan Atlantique 2 femelles du même groupe, dont l'une possédait des œufs. La découverte d'une femelle mature nous a permis de créer une nouvelle espèce, que nous avons placée dans un nouveau genre, Benthimermis.

Les spécimens juvéniles parasites d'Invertébrés diffèrent entre eux par certains caractères : forme des amphides, longueur et forme de la queue, aspect de la région œsophagienne; il nous est impossible dans l'état actuel des connaissances, et étant donné que nous avons affaire à des femelles juvéniles, d'apprécier la valeur de ces caractères; nous avons donc décidé de les placer tous provisoirement dans le genre Benthimermis, sans créer d'espèce différente pour chaque spécimen. Après avoir présenté l'ensemble des caractères communs que possèdent ces spécimens, nous donnerons donc des figures accompagnées d'une brève description de tous les spécimens entiers que nous avons étudiés, en leur donnant simplement un numéro.

Tous les spécimens sont déposés au Muséum national d'Histoire naturelle (M.N.H.N.).

\section{Bentbimermis bopei n. gen., n. sp.}

Holotype (femelle mûre) : M.N.H.N. 1070 BA.

Paratype (femelle juvénile) : M.N.H.N. 1069 BA.

Campagne Thalassa 1973 (Atlantique nord-oriental) ; prélèvement Z 400 ; 22-X-1973 : $47^{\circ} 33,4^{\prime} \mathrm{N}-07^{\circ} 19,0^{\prime} \mathrm{W}$; $1175 \mathrm{~m}$.

Corps épais, peu aminci aux extrémités; cuticule finement striée transversalement, épaisse d'environ $5 \mu \mathrm{m}$; extrémité antérieure arrondie, munie de 4 courtes soies céphaliques submédianes $(16 \mu \mathrm{m})$; ouvertures amphidiales circulaires, amphides en forme de poche à fond en cul de bouteille; cordes latérales et ventrale munies de grosses cellules hypodermiques glandulaires communiquant avec l'extérieur par de fins canaux qui traversent la cuticule. Chez la femelle mature, des concrétions de taille variable se rencontrent çà et là dans la cavité pseudo-cœlomique (fig. $1, A, B$ ).

Pore excréteur et anneau nerveux invisibles.

Ouverture buccale très petite, s'ouvrant dans un fin canal, long de $10 \mu \mathrm{m}$; œso. phage réduit à un cordon à contenu granuleux de diamètre irrégulier, terminé par une masse glandulaire où l'on distingue au moins deux noyaux cellulaires ; intestin transformé en trophosome, sans communication avec le cordon œsophagien, formé de grandes cellules empilées, riches en granulations de réserve; le trophosome occupe toute la cavité pseudo-cœlomique, sauf au niveau de l'appareil génital où il est très 


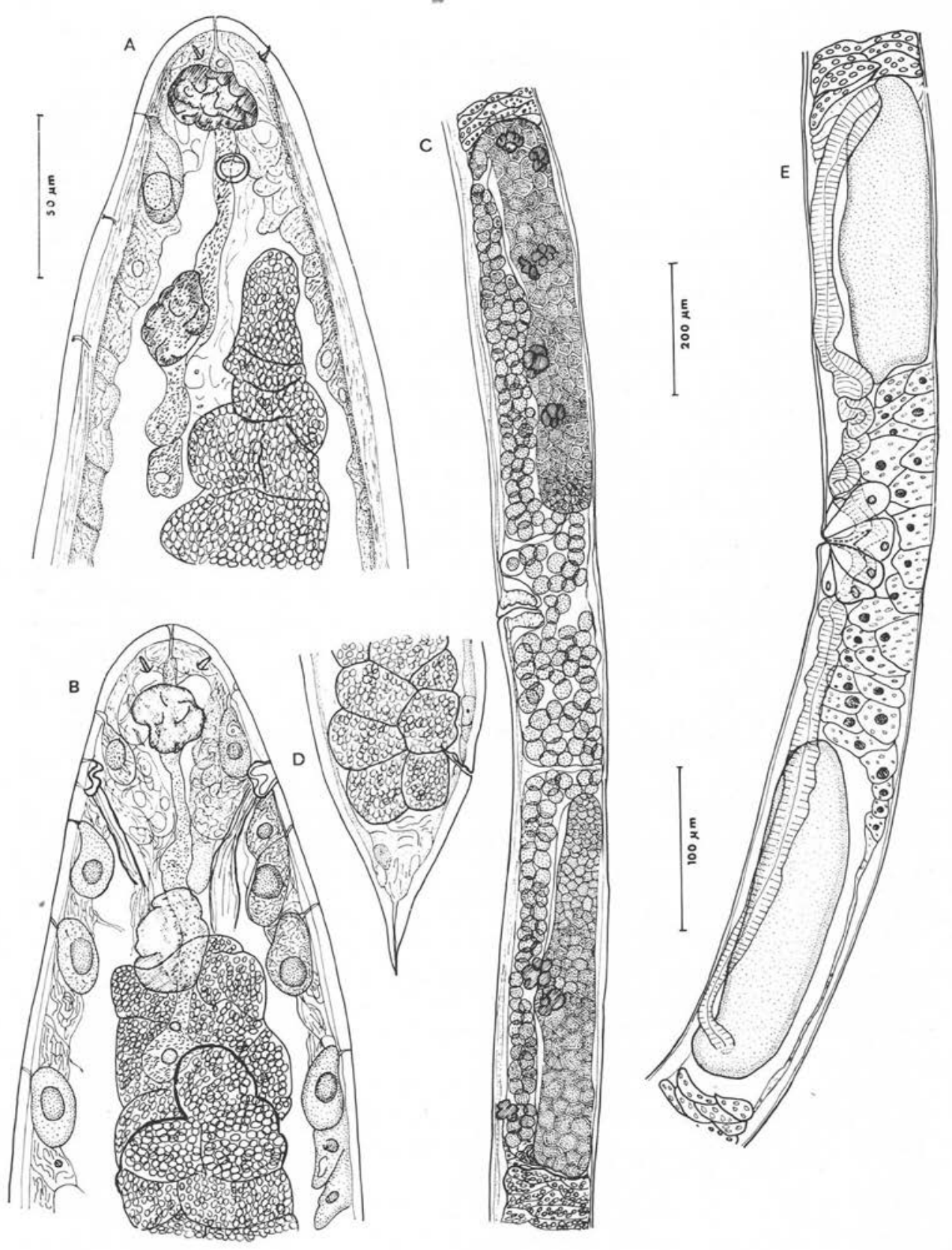

Fig. 1. Benthimermis hopei n.g., n. sp.

A, B, C, D : Holotype. A : extrémité antérieure, vue latérale. B : extrémité antérieure, vue ventrale. C : appareil génital, vue latérale (pour simplifier le dessin, les grosses cellules entourant la vulve n'ont pas été représentées). D : extrémité postérieure, vue latérale. E: appareil génital de femelle juvénile (paratype). A, B : éch. $50 \mu \mathrm{m}$; C, E : éch. $200 \mu \mathrm{m}$; D. : éch. $100 \mu \mathrm{m})$. 
aminci ; dorsalement, il envoie vers l'avant un cæcum qui s'étend le long du cordon œsophagien (fig. 1, A, B). Anus réduit à un petit pore; rectum vestigial, à paroi cuticulaire, sans communication avec le trophosome (fig. 1, D).

Vulve en fente transversale, légèrement antérieure au milieu du corps; vagin à paroi cuticulaire épaisse, dirigé perpendiculairement à la paroi du corps, débouchant dans deux tubes génitaux de directions opposées qui aboutissent à deux gros ovaires récurrents, dont les extrémités distales sont situées au voisinage de la vulve (fig. 1, $C, E)$; chez la femelle holotype, les tubes génitaux sont remplis d'œufs ovales, non embryonnés (fig. 1, C) ; la vulve est entourée par un massif de grandes cellules disposées en rosace autour de l'ouverture (fig. 1, E).

Queue terminée en une pointe effilée, pourvue jusqu'à son extrémité d'un axe cytoplasmique.

Dimensions de la femelle holotype: Longueur: 6,07 $\mathrm{mm}$; largeur maximale : $175 \mu \mathrm{m}$; longueur du cordon œsophagien : $150 \mu \mathrm{m}$; amphides situées à $50 \mu \mathrm{m}$ de l'extrémité antérieure; trophosome débutant à $75 \mu \mathrm{m}$ de l'extrémité antérieure; vulve située à $2,9 \mathrm{~mm}$ de l'extrémité antérieure ; extrémités proximales des ovaires situées respectivement à $2,05 \mathrm{~mm}$ et $2,3 \mathrm{~mm}$ des extrémités antérieure et postérieure du corps ; longueur de la queue : $135 \mu \mathrm{m}$; longueur du rectum : $25 \mu \mathrm{m}$; dimensions des œufs : $60 / 40 \mu \mathrm{m}$.

\section{Spécimens juvéniles parasites d'Invertébrés}

\section{A. - Caractères communs.}

Les spécimens parasites d'Invertébrés sont repliés dans la cavité générale de l'hôte, dont ils occupent en général tout l'espace ; deux spécimens peuvent se trouver dans un même individu-hôte.

Le corps est épais, peu aminci aux extrémités; la cuticule, épaisse de 2 à $8 \mu \mathrm{m}$, est finement striée transversalement; la queue peut être très courte et arrondie ou terminée en pointe; certains spécimens sont munis d'un long appendice caudal (fig. 5, $C$; fig. $7, J$ ). L'ouverture buccale, très petite, est entourée par un cercle de 6 petites papilles (visibles en vue apicale), lui-même encadré par 4 courtes soies céphaliques submédianes (fig. 2, A). Les amphides, relativement éloignées de l'extrémité antérieure (de 30 à $80 \mu \mathrm{m}$ ), ont une ouverture en forme de pore; elles peuvent être tubulaires (fig. $4, A$; fig. $7, A, F, I$ ) ou en forme d'ampoule à contours plus ou moins réguliers (fig. 5, B, E; fig. 6, B, E).

Les coupes histologiques sériées montrent 8 cordes hypodermiques longitudinales : 2 latérales et 2 médianes bien développées, et 4 submédianes réduites, comprenant chacune une seule rangée de cellules (fig. 3, $A, B$ ). Les rangées sublatérales des cordes latérales et médianes sont munies dans la région antérieure de grosses cellules hypodermiques glandulaires communiquant avec l'extérieur par de fins canaux qui traversent la cuticule. Le pore excréteur n'a été observé chez aucun spécimen. 


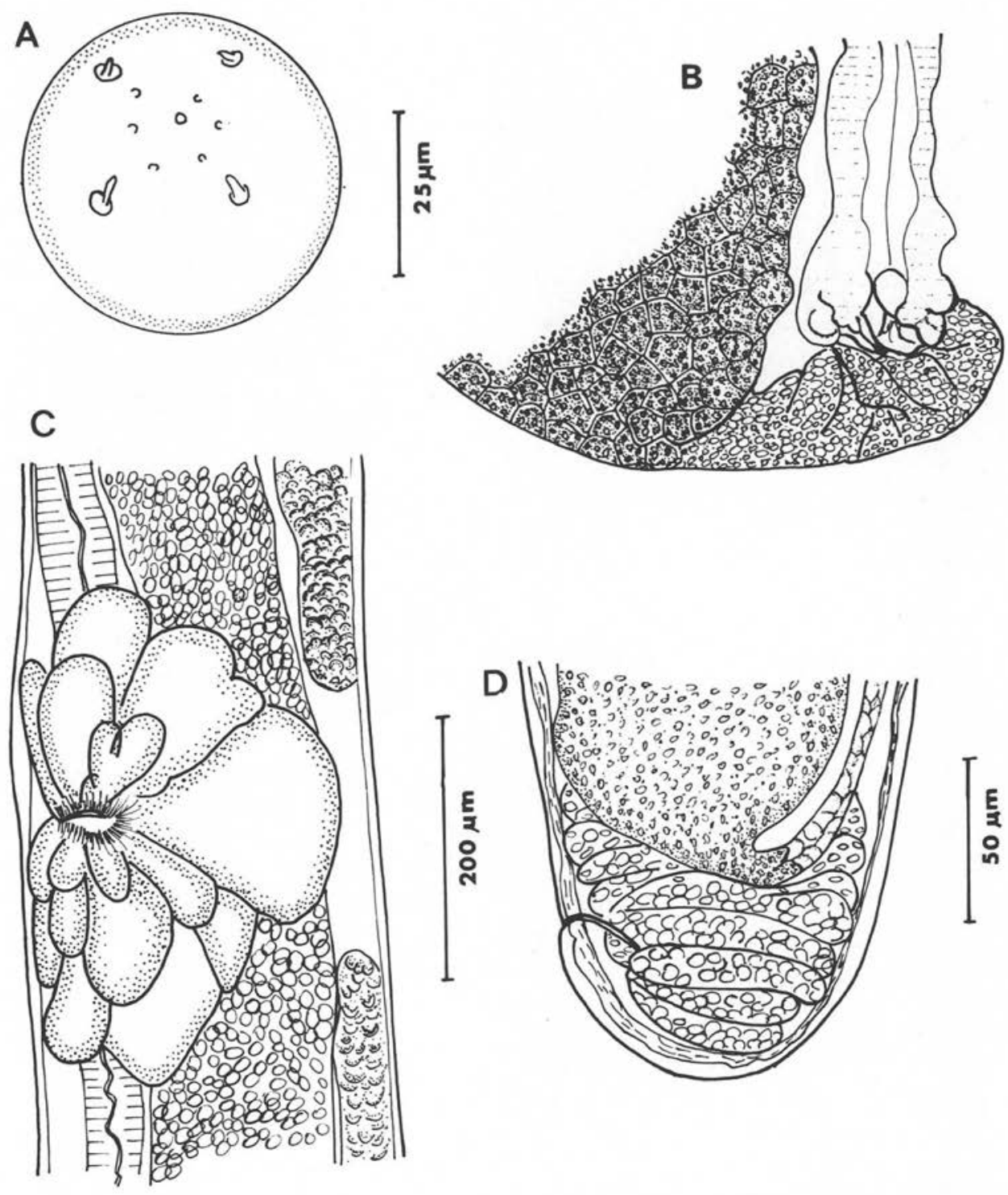

Fig. 2. Benthimermithidae $\mathrm{n}$. fam. (les dessins ont été faits sur des spécimens fragmentaires). A : vue apicale (spécimen parasite d'Isopode de la Mer de Norvège, $\mathrm{n}^{\circ} 1003 \mathrm{BA}$ ). B : jonction ovaire-tube génital (spécimen parasite d'Amphipode de la Mer de Norvège, $\mathrm{n}^{\circ} 1004$ BA). $\mathrm{C}$ : région vulvaire (spécimen $\mathrm{n}^{\circ} 1004 \mathrm{BA}$ ). D : extrémité postérieure (spécimen $\mathrm{n}^{\circ} 1004 \mathrm{BA}$ ) (A: éch. $25 \mu \mathrm{m}$; B : éch. $50 \mu \mathrm{m}$; C, D : éch. $200 \mu \mathrm{m}$ ). 

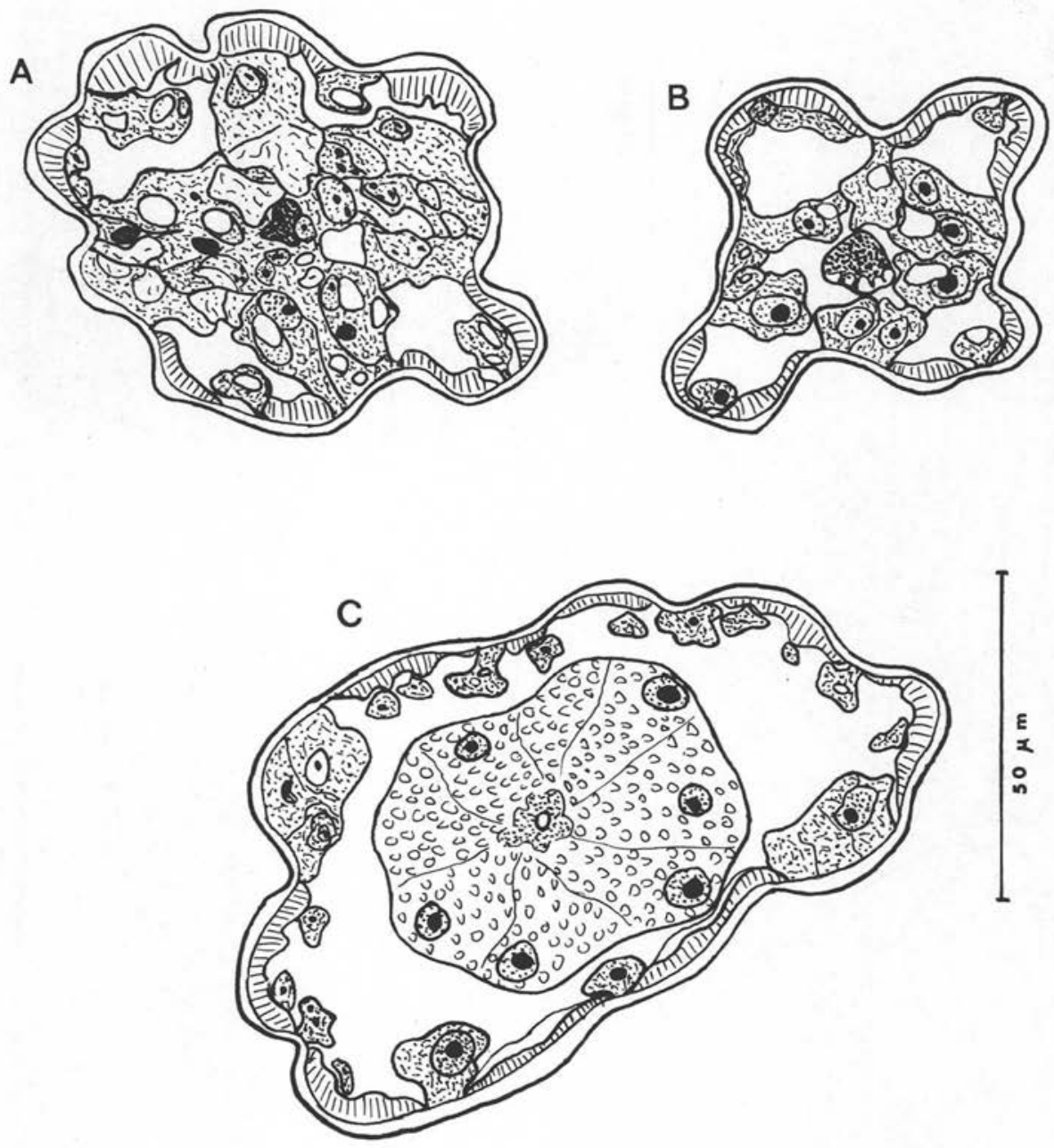

Fig. 3. Benthimermithidae $\mathrm{n}$. fam. (les coupes histologiques ont été faites sur des spécimens fragmentaires). A, B : coupes transversales au niveau de la région œsophagienne (A : spécimen parasite d'Amphipode de l'Atlantique nord-oriental, $\mathrm{n}^{\circ} 997 \mathrm{BA}, \mathrm{B}$ : spécimen parasite d'Isopode de la Mer de Norvège, $\left.\mathrm{n}^{\circ} 995 \mathrm{BA}\right) . \mathrm{C}$ : coupe transversale au niveau du trophosome (spécimen $\mathrm{n}^{\circ} 995 \mathrm{BA}$ ). 


\section{Appareil digestif.}

Le stoma est réduit à un fin canal long de 8 à $15 \mu \mathrm{m}$; l'œsophage, dépourvu de paroi musculaire et de lumière centrale, est représenté par un cordon plus ou moins épais, de longueur variable et de diamètre irrégulier, à contenu granuleux ; à l'extrémité postérieure de ce cordon, existe chez la plupart des spécimens une masse glandulaire qui paraît constituée suivant les cas par une ou plusieurs cellules ; autour du cordon œsophagien, la cavité pseudo-cœlomique est occupée par un tissu riche en vacuoles constitué par le grand développement des cordes latérales et médianes qui se rejoignent au centre et se soudent entre elles (fig. $3, A, B$ ); à l'extrémité antérieure, ce tissu est très peu dense et l'on peut souvent délimiter une zone antérieure constituée de larges cavités séparées par des travées cytoplasmiques (fig. 5 , $A, B, E)$.

Postérieurement à cette région, le corps est occupé par un trophosome qui s'étend jusqu'au niveau de l'anus; il est formé de grandes cellules empilées à cytoplasme riche en granulations; les coupes histologiques montrent que le trophosome porte transversalement des cloisons radiaires, plus ou moins visibles suivant les coupes, séparant une dizaine de cellules disposées autour d'une très petite lumière centrale, et dont les noyaux sont situés à la périphérie (fig. $3, C$ ) ; par endroits, les cloisons radiaires et la lumière centrale deviennent indiscernables et le trophosome présente un contenu uniformément granuleux avec de rares noyaux épars.

Il n'existe pas de communication entre le trophosome et le cordon œsophagien, et celui-ci s'étend quelquefois postérieurement au-delà de la limite antérieure du trophosome (fig. 5, A, D).

L'anus est réduit à un pore ou à une petite fente (fig. 4, C et fig. $7, B$ ); le rectum est généralement entouré par une couche de cellules épithéliales; chez la plupart des spécimens, il n'y a pas de communication entre le rectum et le trophosome, qui se termine d'une manière aveugle légèrement au-dessus ou légèrement en-dessous de l'anus (fig. 2, D); chez quelques spécimens cependant, rectum et trophosome communiquent (fig. 7, $\mathrm{J}$ ).

\section{Système nerveux.}

Chez la plupart des spécimens, des fibres transversales sont visibles en avant de la masse glandulaire située à l'extrémité du cordon œesophagien, correspondant vraisemblablement à l'anneau nerveux (fig. $4, A, B$; fig. $5, D$; fig. 6, A, D).

\section{Appareil génital.}

La vulve, légèrement antérieure ou légèrement postérieure au milieu du corps, est en fente transversale; elle est généralement entourée par un massif de grandes cellules disposées en rosace autour de l'ouverture (fig. 2, C) ; le vagin, à revêtement cuticulaire épais, est dirigé perpendiculairement ou légèrement obliquement à la 


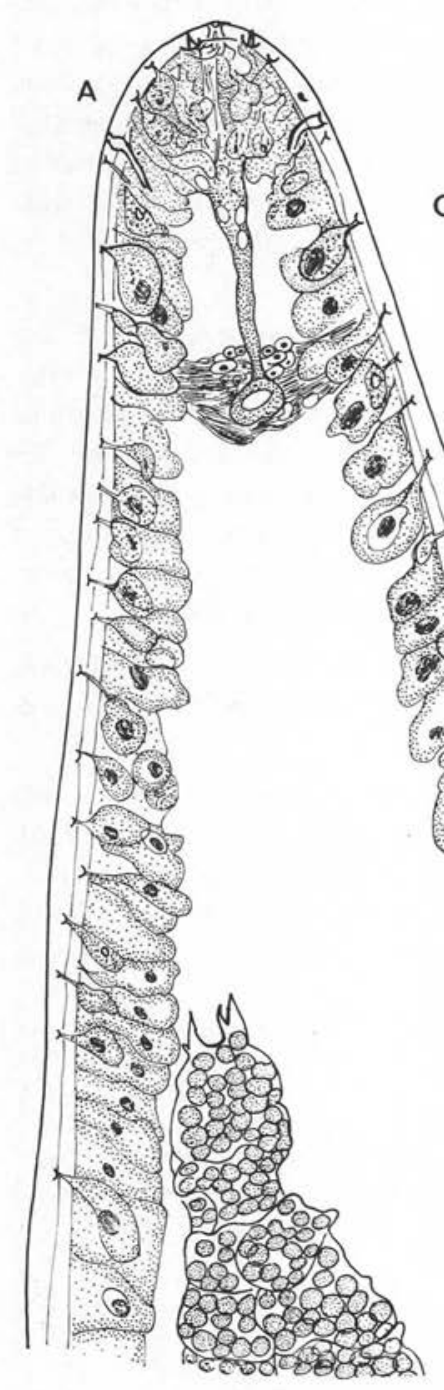

C
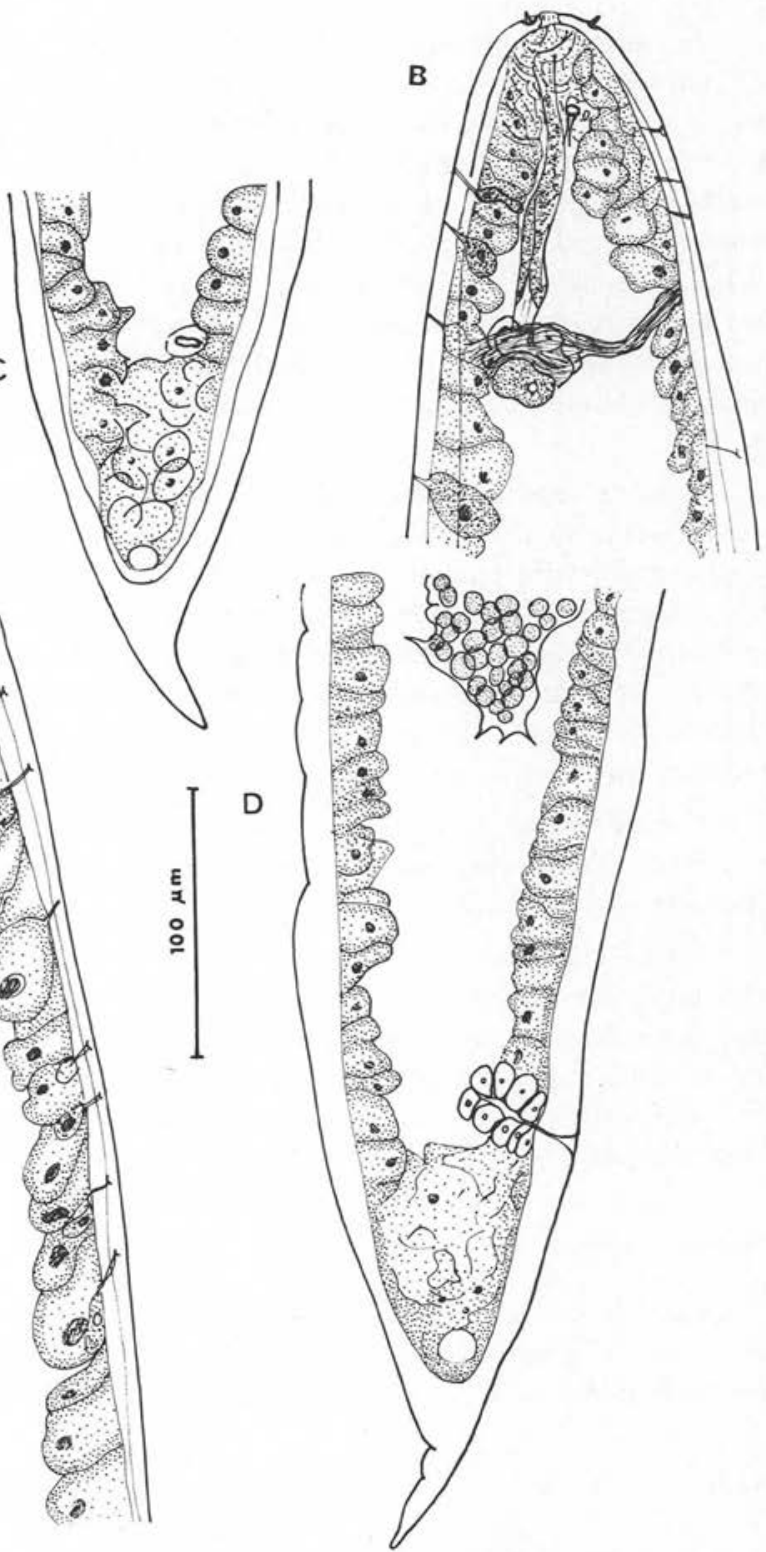

Fig. 4. Benthimermis sp. $\mathrm{n}^{\circ}$ 1. A : extrémité antérieure, vue médiane. B : extrémité antérieure, vue latérale. C: extrémité postérieure, vue ventrale. D: région postérieure, vue latérale. 
paroi du corps (fig. $7, E, H$ ); il s'ouvre dans deux tubes génitaux de directions opposées qui aboutissent à deux ovaires récurrents (fig. 2, B; fig. $7, E$ ).

Chez certains spécimens, l'appareil génital est à un stade précoce de développement, les ovaires sont à l'état d'ébauches piriformes (fig. $7, E$ ) ; chez d'autres, au
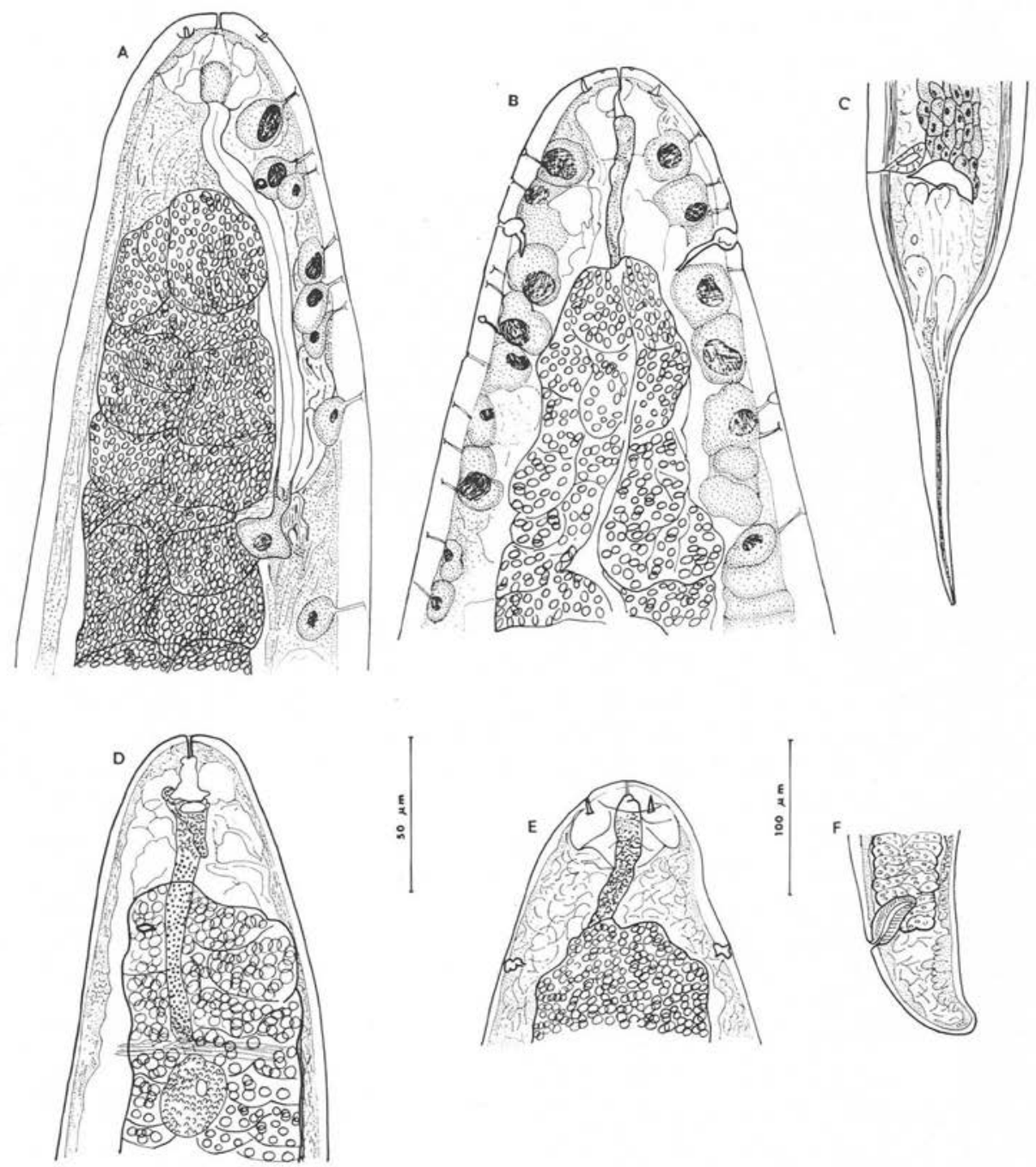

Fig. 5. A, B, C : Benthimermis sp. $\mathrm{n}^{\circ}$ 2. A : extrémité antérieure, vue latérale. B : extrémité antérieure, vue médiane. C: extrémité postérieure, vue latérale. D, E, F: Benthimermis sp. $\mathrm{n}^{\circ} 3$ : D : extrémité antérieure, vue latérale. E : extrémité antérieure, vue médiane. F : extrémité postérieure, vue latérale (A, B, D, E : éch. $50 \mu \mathrm{m}$; C, F : éch. $100 \mu \mathrm{m}$ ). 
contraire, il est très développé et s'étend presque jusqu'aux extrémités du corps (fig. $2, D$ ) ; les ovaires, très longs, ont leurs extrémités distales au voisinage de la vulve (fig. $2, C$ ).

\section{B. - Description des spécimens.}

Les caractères communs au groupe ayant été exposés, nous ne signalerons pour chaque spécimen que leurs caractères particuliers.

Benthimermis sp., $\mathrm{n}^{\circ} 1$ (fig. 4).

M.N.H.N. 1009 BA; hôte: Amphipode (1) ; campagne: Incal (Atlantique Nordoriental) ; prélèvement : DS 01 ; 15-VII-1976; 57059,7’ N-10³9,8’W ; 2091 m.

Longueur : 6,7 mm; largeur maximale : $130 \mu \mathrm{m}$; amphides tubulaires, situées à $40 \mu \mathrm{m}$ de l'extrémité antérieure ; cordon œsophagien étroit, long de $120 \mu \mathrm{m}$; le trophosome débute à une grande distance du cordon œsophagien et se termine bien en avant de l'anus, ceci étant vraisemblablement un artefact dû à la fixation ; vulve située à 3,4 mm de l'extrémité antérieure ; tubes génitaux longs de $1200 \mu \mathrm{m}$ environ, ébauches ovariennes longues de $450 \mu \mathrm{m}$; queue longue de $160 \mu \mathrm{m}$, terminée par une pointe cuticulaire longue de $65 \mu \mathrm{m}$, dépourvue d'axe cytoplasmique.

Benthimermis sp., $\mathrm{n}^{\circ} 2$ (fig. 5, A, B, C).

M.N.H.N. 1001 BA; hôte : Lepechinella manco, Barnard, 1973 (2) (Amphipode) ; campagne: Thalassa 1970 (Sud du golfe de Gascogne) ; prélèvement : W 357 ; 4-X1970 ; 433, $0^{\prime} \mathrm{N}-2^{\circ} 19,2^{\prime} \mathrm{W}$; $920 \mathrm{~m}$.

Longueur : 10,8 mm ; largeur maximale ; $250 \mu \mathrm{m}$; amphides en forme d'ampoule situées à $55 \mu \mathrm{m}$ de l'extrémité antérieure ; cordon œsophagien étroit, long de $160 \mu \mathrm{m}$, s'étendant le long de la région antérieure du trophosome; trophosome débutant à $65 \mu \mathrm{m}$ de l'extrémité antérieure, coiffant à son extrémité postérieure une large cavité allongée transversalement; vulve située à $5,3 \mathrm{~mm}$ de l'extrémité antérieure; ovaires très allongés, à extrémités proximales situées respectivement à $1 \mathrm{~mm}$ et $800 \mu \mathrm{m}$ des extrémités antérieure et postérieure du corps; queue longue de $260 \mu \mathrm{m}$, terminée par une longue pointe de $180 \mu \mathrm{m}$, traversée jusqu'à son extrémité par un axe cytoplasmique.

Benthimermis sp., $\mathrm{n}^{\circ} 3$ (fig. 5, D, E, F).

M.N.H.N. 998 BA ; hôte : Eudorella intermedia Hansen, 1920 (3) (Cumacé) ; campagne : Incal ; prélèvement : ØS 08 ; 11-VIII-1976 ; 47²9,8’’ N-9³9,2’W ; 4327 m.

(1) La détermination spécifique de l'hôte n'a pas toujours pu être faite, en raison du mauvais état des spécimens.

(2) Nous remercions $M^{\text {me }}$ Bellan (Station Marine d'Endoume) qui a identifié ce spécimen.

(3) Nous remercions M. Bishop (Department of Marine Biology, Isle of Man, Port Erin) qui a identifié ce spécimen. 

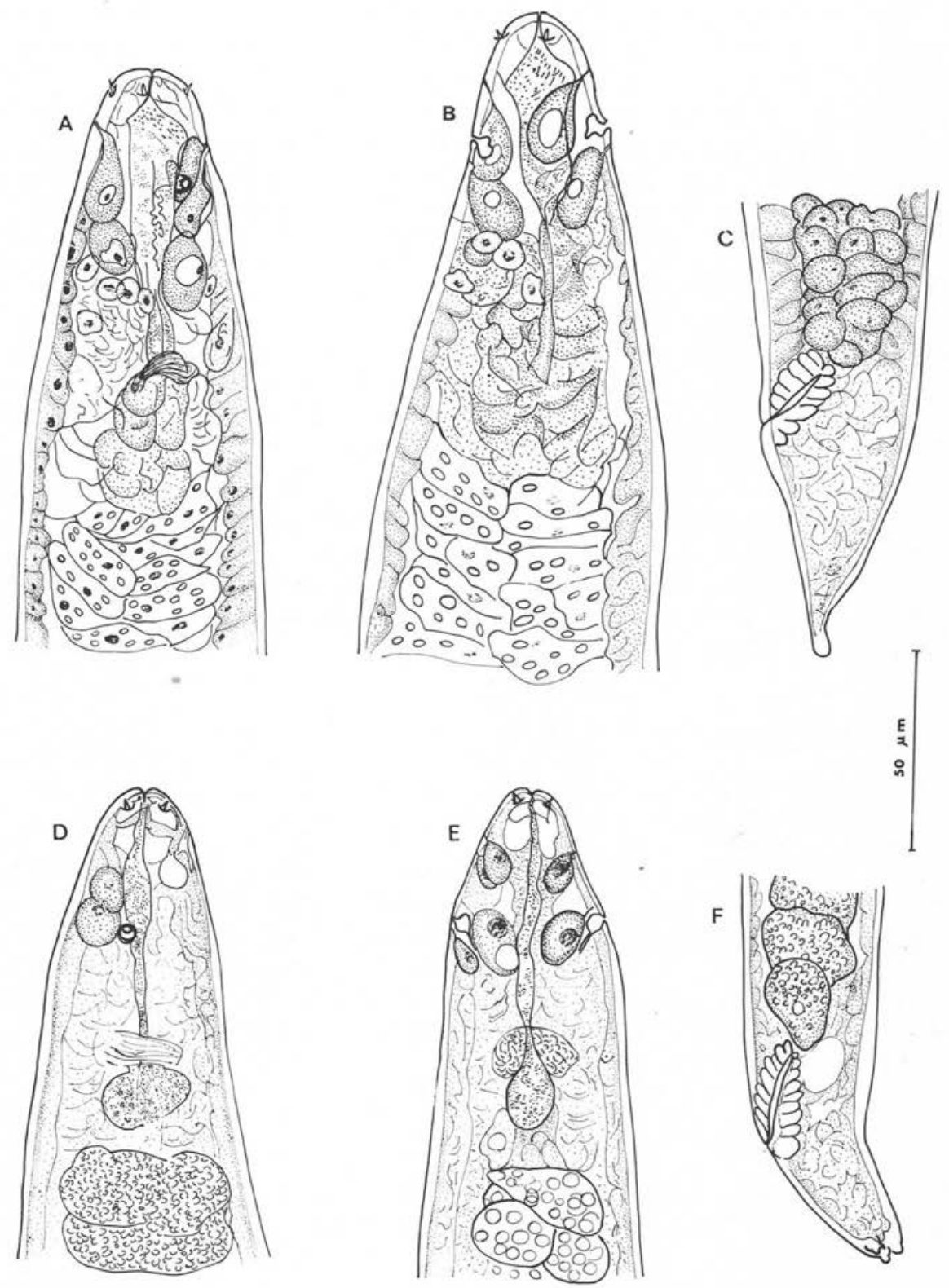

Fig. 6. A, B, C: Benthimermis sp. $\mathrm{n}^{\circ}$ 4. A : extrémité antérieure, vue sublatérale. B : extrémité antérieure, vue médiane. C: extrémité postérieure, vue latérale. D, E, F : Benthimermis sp. $\mathrm{n}^{\circ}$ 5: D: extrémité antérieure, vue latérale. E : extrémité antérieure, vue médiane. F : extrémité postérieure, vue latérale. 
Longueur : 6,4 $\mathrm{mm}$; largeur maximale : $180 \mu \mathrm{m}$; amphides en forme d'ampoule situées à $60 \mu \mathrm{m}$ de l'extrémité antérieure; cordon œsophagien relativement large, long de $100 \mu \mathrm{m}$, s'étendant le long de la région antérieure du trophosome ; à l'extrémité antérieure du cordon œsophagien, on observe une pièce hyaline en forme de bouchon de champagne; trophosome débutant à $50 \mu \mathrm{m}$ de l'extrémité antérieure; vulve située à $3,4 \mathrm{~mm}$ de l'extrémité antérieure ; ovaires très allongés, à extrémités proximales situées respectivement à $400 \mu \mathrm{m}$ et $375 \mu \mathrm{m}$ des extrémités antérieure et postérieure du corps ; queue cônique, longue de $80 \mu \mathrm{m}$, arrondie à l'extrémité et légèrement incurvée dorsalement.

Benthimermis sp., $\mathrm{n}^{\circ} 4$ (fig. 6, D, E, F).

M.N.H.N. 993 BA; hôte : Isopode; campagne : Norbi (Mer de Norvège) ; prélèvement: CP 17 ; 8-VIII-1975; 7330,7’ N-1339,6’W ; $2502 \mathrm{~m}$.

Longueur : 2,7 mm ; largeur maximale : $100 \mu \mathrm{m}$; amphides en forme d'ampoule situées à $30 \mu \mathrm{m}$ de l'extrémité antérieure ; cordon œsophagien étroit, long de $70 \mu \mathrm{m}$; trophosome débutant à $95 \mu \mathrm{m}$ de l'extrémité antérieure; vulve située à $1,4 \mathrm{~mm}$ de l'extrémité antérieure; ovaires très allongés, à extrémités proximales situées respectivement à $425 \mu \mathrm{m}$ et $275 \mu \mathrm{m}$ des extrémités antérieure et postérieure du corps ; queue cônique, longue de $40 \mu \mathrm{m}$, terminée par 3 petits lobes.

Benthimermis sp., no 5 (fig. 6, A, B, C).

M.N.H.N. 1002 BA ; hôte : Tanaïdacé ; campagne : Norbi ; prélèvement: CP 17 ; 8-VIII-1975; 7330,7’N-1339,6’W ; 2502 m.

Longueur : 2,75 mm ; largeur maximale : $100 \mu \mathrm{m}$; amphides en forme d'ampoule situées à $30 \mu \mathrm{m}$ de l'extrémité antérieure; cordon œsophagien long de $70 \mu \mathrm{m}$, très large dans sa partie antérieure ; trophosome débutant à $110 \mu \mathrm{m}$ de l'extrémité antérieure; vulve située à $1,4 \mathrm{~mm}$ de l'extrémité antérieure; ovaires très allongés, à extrémités proximales situées respectivement à $700 \mu \mathrm{m}$ et $550 \mu \mathrm{m}$ des extrémités antérieure et postérieure du corps; qucue cônique, longue de $60 \mu \mathrm{m}$, terminée par un petit appendice arrondi.

Benthimermis sp., $\mathrm{n}^{\circ} 6$ (fig. 7, A, B, C, D, E).

M.N.H.N. 1012 BA ; hôte : Tanaïdacé ; campagne : Véma (Océan Atlantique, nordest des Antilles); prélèvement :' CP 03; 16-XI-1977; $10^{\circ} 47,13^{\prime} \mathrm{N}-42^{\circ} 41,01^{\prime} \mathrm{W}$; $5127 \mathrm{~m}$.

Longueur : 1,8 mm; largeur maximale : $75 \mu \mathrm{m}$; amphides tubulaires situées à $40 \mu \mathrm{m}$ de l'extrémité antérieure ; cordon œsophagien large, long de $60 \mu \mathrm{m}$, prolongé par une grosse masse glandulaire accolée à la région antérieure du trophosome ; trophosome débutant à $70 \mu \mathrm{m}$ de l'extrémité antérieure; vulve située à $1 \mathrm{~mm}$ de 
l'extrémité antérieure; tubes génitaux longs de $150 \mu \mathrm{m}$ environ, aboutissant à de petites ébauches ovariennes piriformes; queue cônique, longue de $50 \mu \mathrm{m}$.

Benthimermis sp., $\mathrm{n}^{\circ} 7$ (fig. $7, F, G, H$ ).

M.N.H.N. 1013 BA ; hôte : Annélide polychète ; campagne ; MD 08 (Océan Indien) ; prélèvement : BB 264 ; 18-IV-1976 ; 4602,0’S-4908,5'E ; $980 \mathrm{~m}$.

Nématode entouré d'une cuticule de mue décollée aux extrémités.

Longueur : 2,2 mm; largeur maximale : $60 \mu \mathrm{m}$; amphides tubulaires, situées à $40 \mu \mathrm{m}$ de l'extrémité antérieure ; limites du cordon œsophagien difficiles à distinguer, surtout dans sa région postérieure ; masse glandulaire terminale et fibres transversales invisibles; trophosome débutant à $110 \mu \mathrm{m}$ de l'extrémité antérieure ; vulve située à $900 \mu \mathrm{m}$ de l'extrémité antérieure; tubes génitaux longs de $150 \mu \mathrm{m}$, terminés par des ébauches ovariennes piriformes; queue longue de $65 \mu \mathrm{m}$, terminée en pointe.

Benthimermis sp., $\mathrm{n}^{\circ} 8$ (fig. 7, I, J).

M.N.H.N. 1015 BA; libre dans les sédiments ; campagne: Norbi ; prélèvement : DS 10 ; 26-VII- 1975 ; 693, $4^{\prime} \mathrm{N}-10^{\circ} 28,6^{\prime} \mathrm{E} ; 2939 \mathrm{~m}$.

Longueur : 8,8 $\mathrm{mm}$; largeur maximale : $200 \mu \mathrm{m}$; amphides tubulaires, situées à $80 \mu \mathrm{m}$ de l'extrémité antérieure ; cordon œsophagien étroit, long de $140 \mu \mathrm{m}$; trophosome débutant à $175 \mu \mathrm{m}$ de l'extrémité antérieure; vulve située à $4,8 \mathrm{~mm}$ de l'extrémité antérieure; tubes génitaux longs de $700 \mu \mathrm{m}$ environ, aboutissant à des ébauches ovariennes piriformes; rectum en communication avec le trophosome; queue longue de $140 \mu \mathrm{m}$, terminée par un long appendice caudal $(100 \mu \mathrm{m})$, pourvu jusqu'à son extrémité d'un axe cytoplasmique.

\section{Discussion}

L'existence de Nématodes marins, libres ou parasites d'Invertébrés, apparentés aux Mermithoidea n'est connue que depuis peu de temps.

Précédemment, deux genres avaient été décrits, sans que leurs affinités avec les Mermithoidea ne soient soupçonnées : d'une part, Ward (1933) avait créé pour un Nématode parasite d'Ophiuride le genre Thalassonema, qu'il n'avait pu attribuer à aucune famille existante ; d'autre part, Hope et Murphy (1969) avaient décrit des mâles d'un nouveau Nématode marin abyssal, Rhaptothyreus typicus, dépourvu apparemment d'œsophage fonctionnel et d'intestin, pour lequel ils avaient créé une nouvelle famille, les Rhaptothyreidae, de position systématique incertaine.

En 1974, Rubtsov et Platanova décrivent deux nouveaux genres, dont les représentants ont été trouvés dans des Echinodermes ou libres dans des sédiments marins ; ils créent pour eux la famille des Marimermithidae, dans laquelle ils placent le genre 

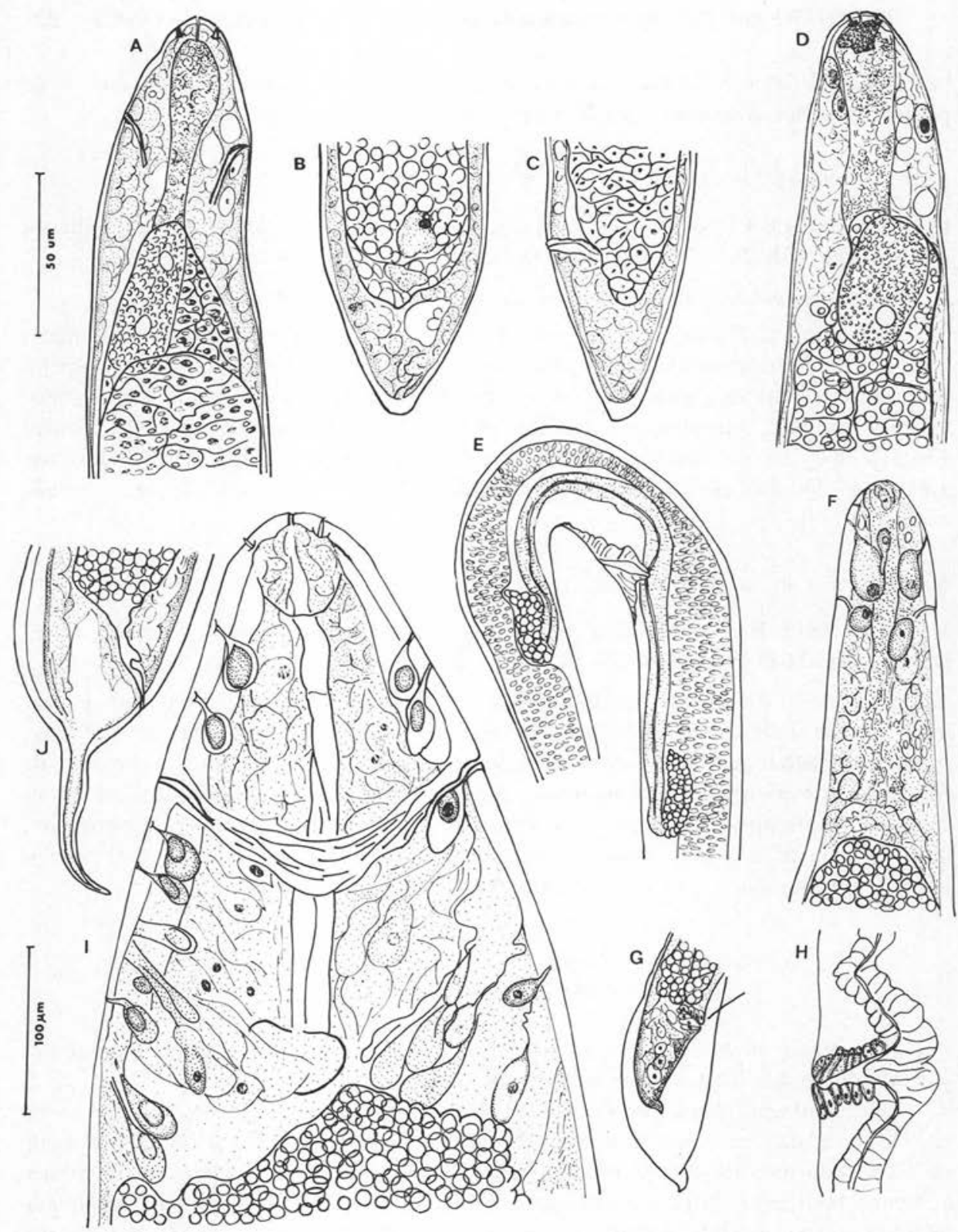

Fig. 7. A, B, C, D, E : Benthimermis sp. $\mathrm{n}^{\circ}$ 6. A : extrémité antérieure, vue médiane. B : extrémité postérieure, vue ventrale. C: extrémité postérieure, vue latérale. D: extrémité antérieure, vue latérale. E : appareil génital, vue latérale. F, G, H: Benthimermis sp. $\mathrm{n}^{\circ} 8$. F : extrémité antérieure, vue médiane. G : extrémité postérieure, vue latérale. $\mathrm{H}$ : région vulvaire, vue latérale. I, J : Benthimermis sp. $\mathrm{n}^{\circ}$ 7. I : extrémité antérieure, vue médiane. J : extrémité postérieure, vue latérale (A, B, C, D, F, H, I : éch. $50 \mu \mathrm{m}$; E, G, J : éch. $100 \mu \mathrm{m}$ ). 
Thalassonema. Rubtsov en 1977 et 1978 place deux nouveaux genres dans cette famille, trouvés l'un dans une Astéride et l'autre libre.

En 1977, Hope signale la découverte dans des stations marines abyssales, à côté des mâles de Rhaptothyreus typicus, d'une population de Nématodes parasites de Crustacés ou libres dans les sédiments, dépourvus de tube digestif, et qui, d'après leur morphologie et leur vie parasitaire, doivent être apparentés aux Mermithoidea ; ces Nématodes sont représentés par de nombreuses femelles et quelques mâles appartenant à plusieurs espèces, dont aucune n'est étroitement apparentée à Rhaptothyreus ; quelques spécimens semblent, par leurs caractères, appartenir à la famille des Mermithidae, tandis que les autres ne peuvent être attribués à cette famille telle qu'elle est actuellement définie. Ces derniers Nématodes ne sont pas décrits en détail, mais les quelques caractères morphologiques signalés par Hope (stoma et œsophage vestigiaux, existence d'un trophosome), ainsi que l'identité des hôtes, nous fait penser qu'ils appartiennent au même groupe que nos spécimens.

Le genre Benthimermis possède en commun avec les Marimermithidae et les Mermithidae l'existence d'un intestin transformé en trophosome et a vraisemblablement une biologie comparable, avec une vie parasitaire limitée aux stades juvéniles ; cependant, il s'oppose à ces deux familles par la structure œsophagienne: l'œsophage est atrophié chez Benthimermis; chez les Marimermithidae, il est bien développé, avec canal central et paroi musculaire; chez les Mermithidae, la paroi musculaire a disparu, mais les glandes œsophagiennes sont organisées en stichosome.

Il possède en commun avec les Rhaptothyreidae un œsophage atrophié, mais par ses amphides de forme simple, il ne peut être placé dans cette famille, qui est définie par l'énorme développement et la structure complexe des amphides.

Il nous paraît donc nécessaire d'isoler le genre dans une nouvelle famille, les Benthimermithidae.

Le degré d'affinité qui existe entre ces différentes familles est difficile à préciser : les Marimermithidae sont les plus proches des Enoplida libres par la structure de l'œsophage. Nous supposons qu'ils ont donné naissance, d'une part, aux Benthimermithidae et aux Rhaptothyreidae, qui ont évolué dans le milieu marin et, d'autre part, aux Mermithidae qui se sont adaptés aux Invertébrés d'eau douce et du sol.

\section{Benthimermithidae n. fam. Diagnose:}

Mermithoidea sensu Andrássy, 1974. Extrémité apicale munie d'un cycle interne de 6 petites papilles et de 4 soies céphaliques submédianes. Ouvertures amphidiales en forme de pore, amphides tubulaires ou en forme de poche. Cordes longitudinales au nombre de 8 . Cordes latérales et médianes munies de grosses cellules hypodermiques glandulaires. Stoma réduit à un fin canal. EEsophage atrophié, représenté par un cordon à contenu granuleux terminé par une masse glandulaire constituée d'une ou plusieurs cellules. Intestin transformé en trophosome. Anus et rectum vestigiaux. Vulve à peu près médiane. Appareil génital femelle amphidelphe, deux ovaires récurrents. 
Femelles juvéniles parasites d'Invertébrés marins, femelles adultes libres dans les sédiments, mâles inconnus.

Genre-type : Benthimermis n. gen. Caractères de la famille.

Espèce-type : Benthimermis hopei n. sp.

\section{Bibliographie}

Andrassy I.: Evolution as a basis for the systematization of Nematodes. Pitman Publishing, 1976, London, San Francisco, Melbourne, 288 p.

Hope W. D. : Gutless Nematodes of the Deep-Sea. Microfauna Meeresboden, 1977, 61, 307-308.

Hope W. D., Murphy D. G. : Rhaptothyreus typicus n. g. n. sp., an abyssal marine nematode representing a new family of uncertain taxonomic position. Proc. Biol. Soc. Washington, 1969, 82, 81-92.

Rubtsov I.A.: A new genus and species of parasitic Nematode, Ananus asteroidus (Nematoda, Marimermithidae), from the Asteroid Diploperaster perigrinator. Bull. Mus. Nat. Hist. Nat., Paris, 3ㅜ sér., 1977, $\mathrm{n}^{\circ}$ 496, Zool. 345, 1113-1117.

Rubtsov I. A.: A new marine nematode Acronema antarcticum gen. et sp. n. Zoologicheskii Zhurnal, 1978, 57, 1573-1575 (en russe, résumé anglais).

Rubtsov I. A., Platanova T. A.: A new family of marine parasitic Nematodes. Zoologiches. Zhurnal, 1974, 53, 1445-1458 (en russe, résumé anglais).

Ward H. B. : On Thalassonema ophioctonis, a nematode parasitic in the brittle star Ophiocten amitinum. J. Parasitol., 1933, 19, 262-268. 